\title{
La evaluación como proceso de aprendizaje: una necesidad del presente, una inversión de futuro
}

\author{
Evaluation as a learning process: \\ a present-day necessity, a future investment
}

\author{
Sara de la Rica Goiricelaya \\ Lucía Gorjón García \\ Imanol Lizarraga Álvarez \\ Fundación ISEAK
}

\section{Resumen}

En la era del Big Data, una verdadera cultura de la evaluación de las políticas públicas se muestra al alcance de la mano. En el presente documento se plantea el papel que jue ga la evaluación de políticas públicas en la mejora de las administraciones y la resolución de algunos de los retos socioeconómicos más acuciantes para nuestras sociedades. Se incide asimismo en el gran salto adelante que supondría una mejora del uso de las bases de datos administrativas para la evaluación. De modo ilustrativo, se presenta un estudio de caso para una mejor comprensión de los beneficios potenciales y tangibles de estas inversiones en apertura y conocimiento.

Palabras clave: evaluación, políticas públicas, retos sociales.

Clasificación JEL: I31, I38, D31, D63.

\section{Abstract}

With the rise of Big Data, a genuine culture of evaluation has become within reach. This article discusses the importance of public policy evaluation in the improvement of public administrations and the resolution of some of our societies' most urgent socioeconomic concerns. Additionally, it underlines the enormous leap forward that an increase in the utilization of administrative data for evaluation would imply. Finally, to illustrate the potential and tangible benefits of those investments in openness and knowledge, a case study is presented.

Keywords: evaluation, public policy, social challenges. 


\section{Introducción}

El debate sobre la evaluación de políticas públicas se ha rearmado en los últimos años, situándose entre las principales prioridades de numerosos gobiernos e instituciones a todos los niveles. Detrás de este creciente interés se encuentran la mejora de la computación, de las bases de datos y de las técnicas apropiadas para una evaluación precisa, así como una mayor concienciación acerca del buen uso de los fondos públicos en aras de una mayor transparencia y rendición de cuentas hacia la ciudadanía. Las posibilidades para perseguir una estrategia de evaluación ambiciosa e innovadora son así mayores que nunca.

Todo programa o política pública se pone en marcha con la intención de resolver problemas y paliar necesidades de la sociedad a la que concierne. No obstante, sin un seguimiento preciso de sus objetivos y resultados, cualquier intervención corre el riesgo de fracasar en su cometido. Sin soluciones adecuadas, los problemas se enquistan y complican. Más allá de buenas intenciones, urgen buenas actuaciones.

La evaluación de políticas públicas se ha popularizado en los últimos años como una forma de abordar esta necesidad. Nos permite guiar el buen funcionamiento de las políticas, destacando las buenas prácticas y advirtiendo de los puntos a mejorar. De este modo, aporta conocimiento útil para la toma de decisiones, mejorando la eficacia y la eficiencia de los programas. En esta época de grandes retos sociales y con una ciudadanía cada vez más concienciada, la exigencia ante las intervenciones públicas es notable.

Para una definición más formal del concepto de evaluación de políticas públicas, nos remitimos aquí a la otorgada por el Ministerio de Política Territorial:

La evaluación de políticas públicas es un proceso sistemático y razonado de generación de conocimiento, a partir de la recopilación, análisis e interpretación de información, encaminado a la compresión global de una intervención pública -sea esta una política, plan, programa o norma-, para alcanzar un juicio valorativo, basado en evidencias, respecto de su diseño, puesta en práctica y efectos (resultados e impactos). (IEPP, 2021)

Como se indica, la evaluación puede enfocarse en cualquiera de las numerosas fases de determinado programa o estudiar el mismo de forma íntegra. Así, una pertinente evaluación ayuda a reconocer si los objetivos planteados están bien definidos, si el diseño del programa se ajusta a estos y si los resultados son satisfactorios.

En este sentido, la evaluación debe verse como una inversión: permite racionalizar el gasto de las diversas administraciones públicas, mejorando su eficacia, eficiencia y flexibilidad; respecto a la ciudadanía a la que sirve, dota a los cuerpos públicos de una mayor transparencia y rendición de cuentas. Mejores actuaciones acercan las instituciones públicas a la sociedad. Adicionalmente, en un entorno cambiante y complejo, las buenas decisiones han de asentarse en la evidencia, subrayando así el papel de la evaluación como aliada natural de la acción pública. En definitiva, la 
cultura de la evaluación es un paso más en la dirección hacia una sociedad de compromiso, abierta al aprendizaje y a la evidencia.

Por otra parte, ninguna evaluación es viable sin buenos datos. La información rica y completa es el mejor recurso de unas administraciones ambiciosas en sus objetivos. A la respuesta de cómo evaluar cabe dar, por tanto, una respuesta corta y directa: reuniendo datos suficientes, primero, y utilizando las técnicas adecuadas, en segundo lugar. Partiendo de estas dos premisas, prácticamente cualquier programa o política es potencialmente evaluable, bien de forma externa, bien internamente.

En el presente estudio se remarca el papel de la evaluación como un aprendizaje continuo, dinámico y con gran potencial para mejorar la acción pública. En primer lugar, se explicará brevemente el concepto de evaluación, dando ligeras pinceladas sobre su importancia y potencial. A continuación, se debatirá sobre el posible alcance de la evaluación -qué se puede evaluar y cómo hacerlo-. Se mostrará el modelo de evaluación integral, que permite estudiar cualquier política o programa desde su fase de diseño hasta los resultados, pasando por la implementación y el seguimiento. En tercer lugar, se introducirá en el debate la importancia del uso de bases de datos administrativos para la evaluación, haciendo especial hincapié en registros administrativos, en particular en las bases de la Seguridad Social. Para ilustrar la aplicación de lo anteriormente expuesto, se presenta en el cuarto apartado un estudio de caso reciente donde se evaluó el contrato en prácticas profesionales en España. Se cierra el estudio con unas breves conclusiones y recomendaciones sobre el camino a tomar.

\section{Qué y cómo evaluar: la evaluación integral}

Con datos suficientes, cualquier intervención pública es evaluable, desde el impacto de un programa de empleo para determinado colectivo hasta una estrategia de lucha contra la pobreza. Para ello, es preciso abordar una inversión inicial en aprendizaje, que permitirá ahorrar cantidades de tiempo, dinero y esfuerzos potencialmente importantes en un futuro. No obstante, el principal y mayor objetivo debería ser siempre aprender sobre las intervenciones realizadas, sobre su alcance, sus limitaciones y sus posibles mejoras. La evaluación puede extenderse a cualquier ámbito de actuación (también privado) que sea medible, como estrategias de gestión o mejoras organizativas. No obstante, es en las políticas públicas, que abordan necesidades sociales crecientes con recursos limitados, donde la evaluación tiene una repercusión de mayor calado (AEVAL, 2010).

Si bien los métodos de evaluación son numerosos, la evaluación integral se considera la más completa y profunda. Se trata de un procedimiento que persigue una comprensión más amplia de las distintas fases en que se desarrolla un programa o política y una identificación exacta de los problemas que puedan llevar a una desviación de los resultados buscados. En este sentido, en lugar de centrarse exclusivamente en la pertinencia de la política en cuestión o en sus resultados, estudia todas y cada 
una de las fases, desde el diseño hasta el impacto, pasando por la implementación y el seguimiento.

Las evaluaciones integrales pueden comenzar antes de la implementación del programa (ex ante), mientras este se ejecuta (intermedia) o al concluir (ex post). Cada enfoque pone el acento en una de las fases de la evaluación, si bien la única diferencia radica en el momento en que se aborda la misma. Mientras que la evaluación intermedia o ex post solo permiten la corrección de errores para futuras implementaciones del mismo programa o similares (por realizarse con el programa ya en marcha), la evaluación ex ante posibilita la detección temprana de puntos débiles y la resolución dinámica de problemas sobre la marcha. En efecto, cuanto antes se comience la misma, antes se podrán corregir los posibles fallos de diseño que pudieran poner en peligro los resultados demandados. Si bien tradicionalmente las evaluaciones intermedias y ex post han estado más extendidas, una buena cultura de la evaluación debería dar mayor valor a las evaluaciones ex ante, que permiten un mejor diseño de políticas públicas y una corrección pronta de problemas de base (AEVAL, 2010).

Como se explicará en las secciones siguientes, la evaluación integral persigue responder diferentes preguntas en cada estadio de la política. En la primera etapa de la evaluación, las cuestiones más relevantes radicarán en la pertinencia del programa y la credibilidad de los resultados esperados. En la segunda etapa, se pondrá el acento en si la implementación está poniéndose en práctica de la forma planeada; en la tercera, se estudiará si los resultados medibles están a la altura de los objetivos planteados o si bien la política tuvo unas consecuencias inesperadas y posiblemente indeseadas. Finalmente se analizará el impacto de la política en cuestión.

\subsection{Las cuatro etapas de la evaluación integral}

Como se introdujo previamente, la evaluación integral sigue una metodología que consta de cuatro etapas, donde se estudian cada una de las fases del ciclo de una política o programa: diseño, implementación, resultados e impacto. Para cada una de las fases se plantea una serie de preguntas y subpreguntas, hipótesis que se tratan de confirmar.

Primera etapa: el diseño es el primer paso en la elaboración de políticas o programas. Se eligen aquí los objetivos a cumplir, se establece la cadena lógica que llevará a su consecución y se dota al conjunto de un marco estratégico con plazos fijos y metas cuantificables. La evaluación de esta fase inicial permite detectar de forma anticipada estrategias desenfocadas y objetivos confusos. Si se evalúa el diseño de un programa aún no implementado, una intervención temprana favorece su refinamiento y reorientación (Instituto para la Evaluación de Políticas Públicas, 2020a).

Son varios los criterios a aplicar a propósito del diseño. En primer lugar, debe haber un diagnóstico del contexto socioeconómico donde se aplicará la política en 


\section{FIGURA 1}

\section{ETAPAS Y CRITERIOS DE LA EVALUACIÓN INTEGRAL}

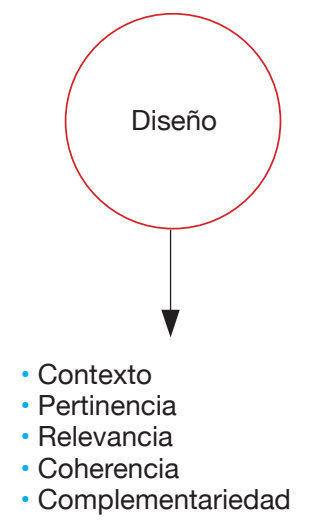

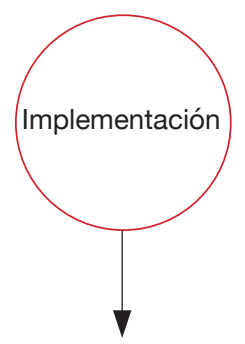

- Gestión

- Presupuesto

- Eficacia

- Cobertura

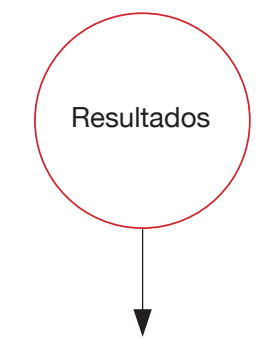

- Eficacia en resultados

- Eficiencia

- Equidad

- Satisfacción

- Sostenibilidad

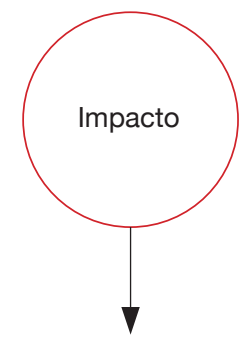

- Impacto

- Contrafactuales

- Microsimulaciones

- Coste-Beneficio

FUENTE: Elaboración propia.

cuestión. Sin conocer el entorno socioeconómico, así como las fortalezas y debilidades existentes, la política corre el riesgo de estar alejada de los problemas y posibilidades reales. Para este ejercicio de contextualización se puede recurrir tanto a bases de datos públicamente disponibles a través de instituciones como los Institutos de Estadística nacionales o regionales, como a registros administrativos, que aportan una información rica en detalle sobre la población en la que intervenir. Segundo, una vez fijado el entorno, cabe realizar un análisis de pertinencia. Las condiciones cambiantes pueden provocar que los objetivos inicialmente fijados queden alejados de los problemas reales. En tercer lugar, ha de estudiarse la relevancia de la política en cuestión. Para ello, se analizará su importancia en la resolución de problemas y se comparará con otras políticas de similares intenciones. El objetivo es la búsqueda de las medidas con capacidad tractora que mayor impacto tengan, alcanzando así en mayor medida los objetivos. Cuarto, se debe hacer hincapié en la coherencia del programa, estudiando los recursos -tanto presupuestarios como humanos-, las medidas que se implementarán y la cadena lógica que generará los resultados. Un programa con objetivos más específicos, financiación suficiente y una estrategia coherente es preferible a uno con ideas más amplias y vagas sobre cómo se conseguirán los objetivos deseados. En quinto y último lugar, se debe atender a la posible complementariedad con programas que afecten a las mismas necesidades y objetivos, con intención de esquivar duplicidades.

Más allá de los anteriores análisis, el uso de microsimulaciones puede ser de especial interés para comprender desde la etapa inicial si el diseño de la política llevará a los resultados esperados. Para ello, pueden realizarse pruebas piloto, donde algunas personas reciban la intervención pública de forma experimental, sirviendo su comportamiento y los resultados observados para determinar la viabilidad de la política, 
estimar el coste de su puesta en marcha y simular los efectos de la acción a mayor escala, siempre bajo determinados supuestos que deben estar razonados en estudios académicos previos o un análisis riguroso del entorno en que operar. Las microsimulaciones no solo permiten estimar el coste de la política, sino también identificar el número óptimo de usuarios potenciales o priorizar aquellas acciones con efectos positivos más significativos.

Segunda etapa: tras el periodo de diseño, la siguiente etapa en el ciclo es la implementación. La evaluación en esta fase se centra en la operativa y organización de la puesta en marcha y seguimiento del programa. Es de especial interés una verificación exhaustiva de la ejecución de los fondos presupuestarios destinados al programa, así como de la gestión y funcionamiento interno del mismo. Las dificultades para gastar los fondos en el lugar adecuado o la escasez de personal pueden hacer descarrilar programas perfectamente válidos. A este respecto, deben estudiarse las posibles barreras de cualquier índole que entorpezcan el programa. La selección de personal del perfil apropiado para las tareas a realizar es asimismo crucial. La calidad de la información que se esté recogiendo es, no obstante, la preocupación primordial en estas etapas iniciales. Solo mediante bases de datos suficientes será posible la evaluación posterior de resultados e impacto. Tras la evaluación del funcionamiento del programa, se debe atender a la eficacia en cuanto a la correcta consecución de fases, procedimientos y objetivos intermedios (con independencia del coste de ejecución). Para ello, se evalúan el grado de cumplimiento y la consumación en los plazos establecidos. Unido al anterior criterio, por último, se encuentra el análisis de cobertura (Instituto para la Evaluación de Políticas Públicas, 2020b). Mediante el mismo se pretende contabilizar tanto la proporción de la población objetivo que ha sido alcanzada por la política -y compararla con los objetivos iniciales-, como la proporción de personas fuera del grupo objetivo que participa en el programa -una medida de lo bien enfocada que está una política y del modo ineficiente en que se gastan los fondos públicos.

Tercera etapa: con el programa o política a pleno rendimiento, cabe remitirse a la evaluación de resultados, la tercera etapa de la evaluación integral, donde se intenta responder hasta qué punto han sido logrados los objetivos de la política o programa. En esta etapa, no se busca cuantificar específicamente el posible impacto de la política, sino analizar si se han generado los resultados esperados. Por definición, se trata de una evaluación ex post. Son cinco los criterios a seguir en la evaluación de resultados: la eficacia en resultados, la eficiencia, la equidad, la satisfacción y la sostenibilidad. La eficacia en resultados hace referencia al grado de cumplimiento del programa. La eficiencia, mientras, se refiere a la cantidad de recursos empleados para conseguir (o perseguir) los objetivos iniciales. Equidad y satisfacción, por otro lado, se remiten a la igualdad de trato entre los diversos grupos y su conformidad con los resultados. Finalmente, la sostenibilidad del programa debe garantizar su autonomía para mantener los resultados en el tiempo (Instituto para la Evaluación de Políticas Públicas, 2020c). 
Cuarta etapa: el último paso en la evaluación integral de una política es la evaluación de impacto. En este ciclo, se trata de atribuir resultados específicos a las acciones emprendidas dentro de la política, cuantificarlos y calibrar el éxito o fracaso en la consecución de objetivos. En definitiva, en la evaluación de impacto se busca una relación causal entre el programa planteado y los resultados obtenidos. Esta última fase de evaluación se ilustrará mediante un caso práctico en el apartado cuarto del presente estudio. Sin una base de datos adecuada, la evaluación de impacto resulta del todo imposible. Por ejemplo, en la evaluación de un programa de inserción para jóvenes, se debe disponer de datos sobre características sociodemográficas de beneficiarios y no beneficiarios, características sobre contratos y condiciones laborales previas, intermedias y posteriores, etcétera. Estos datos podrían permitir computar el impacto específico de la política en su inserción laboral, así como observar qué grupos socioeconómicos se ven más beneficiados y dónde se podría mejorar (AEVAL, 2010).

La evaluación de impacto puede realizarse bien mediante la construcción de contrafactuales, bien a través de microsimulaciones. Óptimamente, el impacto podría computarse comparando los resultados bajo la aplicación de la política y los resultados sin la misma. No obstante, en la vida real solo se observa la primera. Por ello, para ver el impacto de la política en el grupo de tratamiento (los participantes) se requiere de un grupo de control con quien poder comparar. El grupo de control debe tener unas características poblacionales lo más similares posibles al grupo de tratamiento, de tal modo que su única diferencia radique en la participación o no participación en el programa a evaluar. Comparando ambos y controlando por sus características puede estimarse de forma precisa y rigurosa el impacto real de la política. La evaluación de impacto puede ser experimental o cuasi-experimental según el método de selección de grupos de tratamiento y control. Respecto a la primera, se refiere a la realización de un experimento controlado, donde se selecciona aleatoriamente a participantes y no participantes. Cuando la población objetivo es pequeña, la evaluación experimental puede realizarse tomando a todos los potenciales individuos como muestra; cuando la población es grande, se toma una muestra representativa del grupo general. Las evaluaciones cuasi-experimentales, en cambio, se emplean cuando los experimentos son imposibles o moralmente reprobables -por ejemplo, cuando se necesita excluir a personas de la participación en un programa de vuelta al empleo para poder compararlos con personas que sí acceden-. En esta ilustración, los propios desempleados serían libres de elegir su participación o no en el programa, pudiendo llevar a problemas de sesgo de selección (donde las características de grupo de tratamiento y control fueran diferentes). Existen diversos métodos cuantitativos comúnmente extendidos, como el control de diferencias en diferencias, el emparejamiento o la regresión discontinua, que requieren cierto conocimiento técnico, pero aportan resultados robustos muy relevantes.

Siguiendo el proceso de evaluación en cada una de sus etapas se deben identificar las buenas prácticas, descartando o modificando aquellas políticas que por su 
diseño o sus pobres resultados devienen inapropiadas y potenciando las de resultados positivos. La evaluación, no obstante, no es un proceso estático, sino una técnica dinámica en que el aprendizaje y el cambio razonado mejoren continuamente las intervenciones públicas.

\section{La riqueza y potencial de las bases de datos administrativas}

El combustible primordial a lo largo de todas las fases de la evaluación son los buenos datos. Sin medidas cuantificables, cualquier evaluación es inviable. Las fuentes a través de las cuales se pueden obtener los datos necesarios son numerosas: según su origen (de instituciones públicas o privadas), según su método de obtención (datos de registro, de encuestas, de experimentos, cuestionarios...) o según su duración en el tiempo (datos de sección cruzada, longitudinales o series temporales...).

No obstante, la gran riqueza de las diversas administraciones públicas en cuanto a los datos administrativos se refiere las hace protagonistas en la construcción de una cultura de evaluación moderna. Las administraciones recopilan una gran cantidad de datos de forma precisa que podrían ser de excepcional uso para la evaluación de programas públicos. Adicionalmente, las bases de datos administrativas son complementarias a las encuestas o experimentos. Tienen la gran ventaja de que abarcan a toda la población, por lo que los posibles problemas de tamaño que suelen acompañar a muchas bases de datos provenientes de encuestas no existen. El inconveniente es que a menudo no se dispone de toda la información que sería deseable para abordar una evaluación robusta.

Sin embargo, habitualmente no se reconoce su potencial. Más aún, la falta de integración de las diferentes bases de datos -incluso dentro de una misma institución-, dificulta su uso y lastra su utilidad. Sería recomendable una estrategia global que permitiera una mayor armonización entre las distintas fuentes de datos, con códigos y formas de medición comparables e integrables que salvaguarden el anonimato de las personas que aparecen en la base de datos. Dado que cada base de datos surge con un propósito diferente, una fácil integración entre las mismas permitiría unir perspectivas hasta obtener una comprensión más global.

Un buen ejemplo de buenas prácticas sobre el que avanzar sería la Base de Datos Integrada para la Investigación del Mercado Laboral (IDA), realizada por el servicio estadístico nacional de Dinamarca en colaboración con numerosas instituciones y administraciones del país, y que se estudia en mayor profundidad en Brugarolas et al. (2020). El servicio estadístico danés cuenta en total con más de 250 fuentes estadísticas distintas a disposición del público investigador que se pueden combinar según las necesidades del solicitante. Desde datos poblacionales hasta registros de pago de impuestos, cotizaciones a la seguridad social o datos sobre empleados de empresas, las posibilidades son numerosas. Los datos se ofrecen anonimizados y bajo un compromiso de confidencialidad que garantiza la buena fe de ambas partes, con el espíritu del empleo de las numerosas bases de registro público para la 
investigación y la promoción de la evaluación de políticas. Es en este contexto que el servicio de estadística danés ofrece la IDA como una herramienta ágil y de gran utilidad para la investigación. Se trata de una base de datos anual donde se integran informaciones de diversas fuentes administrativas, obteniendo así en una misma base de datos información de los individuos, de los trabajadores y de las empresas. Respecto a los primeros datos, se incluyen abundantes estadísticas sociodemográficas, de residencia, condiciones familiares, sobre su experiencia profesional o los años en desempleo; en cuanto a los trabajadores, se proporciona información sobre sus periodos de empleo, sobre las condiciones laborales, tipo de contrato, jornada o salario hora; finalmente, se ofrecen datos sobre la localización de empresas, el número de trabajadores o la actividad. Huelga destacar la riqueza de esta base de datos, integrada por información ya existente de forma previa, pero que por su dispersión y falta de integración no tenía el potencial que ahora presenta. Adicionalmente, la IDA permite una gran flexibilidad para incorporar bases de datos externas de terceras fuentes, por lo que sus posibilidades son abiertas y numerosas. Kleven et al. (2019), por ejemplo, utilizan la IDA junto con datos de ingresos familiares del Ministerio de Finanzas danés para estudiar el impacto de la maternidad en la participación laboral de las mujeres. En definitiva, una pequeña inversión en recogida e integración de bases de datos dispersas ha permitido una gran expansión de la cultura de evaluación en el país.

Si se quisiera replicar una propuesta similar en España, no habría que empezar de cero. Existen diversas bases de datos muy ricas en información que podrían servir como columna vertebral de un proyecto de integración de bases administrativas ambicioso y de largo alcance. Un ejemplo de interés de base de datos administrativa de uso habitual para la evaluación de políticas -principalmente en el ámbito laboral- es la Muestra Continua de Vidas Laborales (MCVL). Esta base de datos consiste en la vida laboral completa de un $4 \%$ de las personas afiliadas a la Seguridad Social. En particular, la base de datos recoge todos los episodios de empleo y desempleo de asalariados, con las fechas exactas y su duración, así como el tipo de contrato, horas trabajadas o tamaño de la empresa. También recoge información sobre el autoempleo, fecha de creación de la empresa, sector de actividad, ocupación o características sociodemográficas, así como información disponible sobre la base y grupo de cotización de cada una de las personas y las empresas. Su potencial es grande, pero la departamentización de las diversas bases de datos según la administración responsable dificulta notablemente su integración con otros datos sobre empresas o la población activa. Dado que los datos administrativos ya están recopilados para la gestión de la Seguridad Social, poner a disposición de la comunidad científica un método para su integración con otras bases de datos permitiría que se realizaran innumerables análisis de diversa índole en lo referente a la implementación de políticas laborales, de pensiones, de género, etcétera.

En este punto, es necesario destacar el reciente impulso a una estrategia de integración de bases de datos de diversas fuentes administrativas que está siendo liderada por el Instituto Nacional de Estadística (INE), mediante la cual se pretende 
combinar datos del INE con información fiscal del Ministerio de Hacienda, así como de la Seguridad Social. Este interés y esfuerzo muestra que se avanza en el buen sentido, si bien el trabajo a hacer es aún ingente.

En el estudio de caso presentado a continuación, se muestra el impacto de un buen uso de bases de datos para la medición del impacto de una política activa de empleo dirigida, en concreto, al colectivo de personas jóvenes: los contratos en prácticas. Por otro lado, también se explicarán sus limitaciones, así como las posibilidades que podría abrir una propuesta de integración de bases de datos como la IDA de Dinamarca.

\section{Estudio de caso: el contrato en prácticas. Cómo evaluamos los beneficios/ perjuicios del contrato en prácticas}

En anteriores apartados se incidió en la necesidad de entender la cultura de la evaluación como inversión de futuro. Para ilustrar el proceso y sus posibilidades se presenta a continuación un caso práctico de evaluación de una política pública de España, el contrato en prácticas profesionales para personas jóvenes (De la Rica et al., 2020).

En este caso en concreto, nos centramos en la última etapa de la evaluación integral, el estudio de impacto. No obstante, se deberían analizar asimismo tanto el diseño como la implementación y los resultados del contrato en prácticas para obtener una panorámica más amplia. Adelantando las conclusiones que se mostrarán posteriormente, cabe atribuir a un mal diseño e implementación del referido contrato los pobres resultados observados en la evaluación de impacto. Una evaluación precisa en las primeras etapas de la política en cuestión podría así haber evitado algunos de los problemas que se detectan en el estudio de impacto.

Originalmente ideado para impulsar una transición laboral adecuada de las y los jóvenes desde su etapa formativa superior hacia su etapa laboral, mediante el contrato en prácticas la empresa recibe ciertos beneficios fiscales a cambio del contrato de personas jóvenes, de modo que estas adquieran una práctica adecuada a su formación. La práctica profesional debe venir acompañada de una inversión relevante en formación por parte de la empresa, de tal modo que se generen los incentivos adecuados para la transición a la contratación indefinida tras la conclusión de las prácticas. A cambio, las empresas se ven beneficiadas mediante una reducción del $50 \%$ de las cotizaciones a la Seguridad Social por el trabajador (incentivo retirado en 2018), así como de un salario menor al estipulado por el convenio para el puesto en cuestión durante el periodo subvencionable. El contrato en prácticas debe tener una duración mínima de 6 meses y un máximo de 2 años, con jornada completa o parcial, y la retribución no puede ser inferior al $60 \%$ (durante el primer año) o un $75 \%$ (el segundo año) del salario establecido por convenio para un puesto con esas mismas responsabilidades y tareas. 
En definitiva, se persigue una incorporación fluida y rápida de las personas jóvenes al mercado laboral tras el fin de sus estudios, así como una mayor formación por parte de las empresas, generando así los incentivos para fomentar la estabilidad laboral y la formación adecuada de las personas jóvenes trabajadoras.

Para comprobar si los objetivos descritos para este tipo de contrato se cumplen, se evalúan de forma cuantitativa dos dimensiones particulares de este contrato en prácticas: en primer lugar, la permanencia en la empresa tras la extinción del mencionado contrato; segundo, la transición del contrato en prácticas a contrato indefinido, bien sea en la misma empresa, bien en otra. Para ello, se utiliza la referida Muestra Continua de Vidas Laborales (2017). Se trata de una base de datos muy rica en información relativa al historial laboral de una muestra de personas trabajadoras de España, con detalles sobre cada episodio de empleo y desempleo de los individuos incluidos en ella desde el comienzo de su vida laboral hasta el 31 de diciembre de 2017. Para cada episodio de empleo, se especifica el tipo de contrato y la duración del mismo, así como las características sociodemográficas, el nivel formativo, el género o el lugar de residencia.

Para realizar la evaluación, se selecciona de la MCVL a las personas menores de 30 años con estudios de Formación Profesional o universitarios (requisito para firmar el contrato en prácticas) y se los divide en dos grupos, que servirán para hacer las comparaciones y estimar resultados cuantitativos -números interpretables, en definitiva-. El primer grupo, el grupo de tratamiento, lo componen personas jóvenes cuya primera relación laboral fue un contrato de prácticas; el segundo, el grupo de control, lo constituyen jóvenes de mismas características que los anteriores, pero cuya primera relación laboral fue un contrato temporal de más de tres meses (el primer contrato más común entre las personas jóvenes que entran al mercado laboral a través de un contrato temporal). Como se observa, el grupo de tratamiento es aquel que participa en la política a evaluar, mientras el grupo de control lo forman las personas con quienes compararemos las trayectorias laborales de los primeros. $\mathrm{Si}$ los grupos son similares en cuanto a sus características sociodemográficas, pero se observan divergencias en sus trayectorias posteriores, puede inferirse que las diferencias son imputables al impacto de la política en cuestión, el contrato en prácticas. En este sentido, estamos en posición de evaluar los dos objetivos planteados inicialmente: la permanencia en la empresa y la transición a indefinido.

Respecto al primer objetivo, dado que las empresas realizarían una inversión en formación del trabajador, se esperaría que la permanencia tras las prácticas fuera superior (o al menos no inferior) a las personas jóvenes con contrato temporal ordinario (grupo de control). La teoría económica indica que, cuando las empresas invierten en el capital humano de sus trabajadores/as, esperan retenerlos el tiempo suficiente para recuperar su inversión inicial a través de una mayor productividad del trabajador. En contra del espíritu original de la política, no obstante, se encuentra que quienes firman un contrato en prácticas tienen mayor probabilidad de irse de la empresa que aquellas personas jóvenes cuya primera experiencia laboral es un contrato temporal estándar de más de tres meses. Entre las personas jóvenes que 
completan todo el periodo estipulado de prácticas, solo un tercio se queda con un contrato distinto tras la extinción de las prácticas, una proporción similar a la de las personas del grupo de control, que trabajan bajo contrato temporal. En cambio, entre aquellas que rescinden el contrato en prácticas con anterioridad, tan solo un $16 \%$ se queda en la empresa, frente a un $73 \%$ que cambia de empresa y empleo y un $11 \%$ que transita hacia el desempleo. Teniendo en cuenta que más de la mitad de los contratos de prácticas se extinguen fuera del periodo estipulado, la tasa de permanencia resulta notablemente más baja entre las personas jóvenes en prácticas, respecto a aquellas personas con contrato temporal. Es decir, el contrato en prácticas no estaría fomentando la permanencia en la empresa, sino el efecto contrario. Este resultado sería muy consistente con el hecho de que las empresas no estarían invirtiendo en la formación de estos jóvenes y, por tanto, no existiría el incentivo de mantenerlos en su empresa tras las prácticas subvencionadas.

Segundo, respecto a la transición hacia un contrato indefinido, se observa que aquellas personas que permanecen en la empresa tras finalizar el contrato en prácticas tienen una probabilidad un $20 \%$ inferior de conseguir un contrato indefinido tras las prácticas que quienes empiezan con contrato temporal. Por otro lado, entre quienes finalizan el contrato de prácticas fuera del periodo estipulado y se cambian de empresa, la probabilidad de obtener uno indefinido se incrementa en un $15 \%$. De nuevo, si las empresas formaran al/la trabajador/a, tendrían el incentivo de retener a esas personas mediante la oferta de un contrato estable al finalizar las prácticas. En ausencia de formación, sin embargo, la barrera que suele suponer a una empresa el salto desde un contrato de prácticas a uno indefinido se haría efectiva y las empresas preferirían ofrecer la alternativa que pase por la renovación bajo un contrato temporal estándar (algo que está permitido por ley durante otros dos años) o la extinción de la relación contractual. Adicionalmente, se encuentra un efecto indirecto y no esperado del contrato en prácticas y es que la probabilidad de obtener un contrato indefinido es superior entre aquellas personas jóvenes que no acaban el periodo estipulado de prácticas y se marchan de la empresa en cualquier punto intermedio. Así, una interpretación posible es que las personas jóvenes contratadas mediante un contrato de prácticas, conscientes de la falta de formación y la baja probabilidad de transición hacia un contrato indefinido en la empresa utilizarían el contrato en prácticas de manera temporal a la espera de mejores ofertas laborales. Otra posible explicación podría recaer en la exigencia de experiencia previa para muchas ofertas laborales. Así, las personas jóvenes podrían obtener la experiencia requerida a través del periodo de prácticas, con intención de incrementar sus oportunidades en futuros procesos de selección en terceras empresas.

Los anteriores resultados permiten comprender el impacto que el contrato en prácticas ha tenido en los últimos años sobre las carreras profesionales de las personas jóvenes. En una suerte de círculo vicioso, los jóvenes lo utilizan como contrato puente mientras buscan oportunidades laborales en otras empresas, ya que no tienen evidencia de que la empresa quiera retenerles; las empresas, mientras, saben que los jóvenes lo utilizan como empleo provisional, no invierten en su formación y lo 
utilizan como una forma de obtener beneficios fiscales que abaratan el coste laboral. En resumen, el uso que se hace del contrato en prácticas es contrario al espíritu con el que fue creado, incrementando aún más la precariedad laboral de las personas jóvenes.

\subsection{Qué aprendemos}

La evaluación se puede definir sucintamente como un aprendizaje continuo sobre los factores que no se han tenido en cuenta en el diseño de políticas de cualquier índole. Como se ha demostrado en el apartado anterior, el diseño e implementación del contrato en prácticas no previó determinados comportamientos de empresas y trabajadores/as que han llevado a resultados diferentes a los esperados. Las buenas intenciones son necesarias, pero los buenos resultados lo son más.

La evaluación saca a la luz determinados aspectos a cambiar para conseguir un contrato en prácticas/formativo que sea eficaz en su propósito fundamental. Cualquier contrato en este sentido debe llevar aparejados ciertos requerimientos acreditables de formación en el puesto de trabajo que aseguren la implicación formativa de la empresa en la trayectoria profesional del/la trabajador/a. Por otro lado, las personas jóvenes deben ver beneficios tangibles en las prácticas, de tal modo que su compromiso con la empresa lleve a una maximización de la utilidad que la formación pueda aportar a sus aptitudes profesionales. Construir sobre el aprendizaje que aportan evaluaciones como la presentada previamente debería ser una prioridad para cualquier administración o institución que aspire a servir adecuadamente a su papel social.

\subsection{Quéfalta}

Por supuesto, el estudio del contrato en prácticas referido deja numerosas zonas oscuras que los datos no permiten discernir. Una mejora de la gestión de bases administrativas podría permitir suplir esas faltas y alcanzar resultados más precisos, que ayudarían a orientar las políticas públicas hacia una más eficiente consecución de sus objetivos.

Una base de datos que integrara la MCVL con datos sobre empresas, por ejemplo, permitiría conocer de primera mano las diferencias entre las empresas que mejor están empleando el contrato en prácticas y aquellas que no lo usan adecuadamente. Adicionalmente, esta permitiría conocer el perfil de la empresa y el tipo de contrato que ofrece a otros empleados, la importancia de la cultura de la formación o la estabilidad laboral de sus trabajadores/as. Por otro lado, una mayor integración de la MCVL con otras fuentes de datos sociodemográficas o de Hacienda permitiría conocer la evolución de los ingresos de los jóvenes que firmaron un contrato en prácticas, su campo de estudio o su desempeño académico, todo ello vital para una mejor comprensión del mundo laboral que afrontan los jóvenes de hoy. 
En resumen, los datos están ahí, solo se necesita un impulso público atrevido que garantice el ecosistema adecuado para una cultura de la evaluación rica, moderna e inteligente.

\section{Hacia dónde ir}

En un mundo informatizado con una creciente cantidad de datos, donde los grandes retos socioeconómicos y demográficos requieren soluciones cada vez más complejas, las administraciones e instituciones públicas debieran dirigirse hacia una mayor integración y mejor gestión de la gran riqueza que poseen: los datos. Como se mostró con el modelo danés, pequeñas inversiones pueden deparar a medio y largo plazo grandes rendimientos y mejoras de eficiencia notables. Las instituciones españolas cuentan con grandes profesionales que pudieran poner al país a la vanguardia de la transparencia y la rendición de cuentas pública.

Adicionalmente, la gran competencia por los fondos públicos en el contexto de un Estado de Bienestar en proceso de adaptación a la economía globalizada actual requiere una mejora continua de la eficacia y eficiencia con que se asignan los recursos públicos y se diseñan las políticas públicas que permitan un mayor bienestar social. La evaluación integral de políticas es nuestra mejor herramienta para lidiar con problemas que aparentemente carecen de solución, como la debilidad de la infraestructura laboral, los problemas en el sistema educativo o, más recientemente, los puntos flacos detectados en la sanidad y gestión pública a raíz de la pandemia de Covid-19. Además, una cultura de la evaluación que valore la ecuanimidad, la precisión y la imparcialidad es deseable en sí misma, y puede alcanzar un gran consenso en una sociedad crecientemente polarizada.

En conclusión, no deberíamos desaprovechar el gran activo con que cuentan nuestras administraciones e instituciones. Como en la era de los combustibles fósiles lo fue el petróleo, en la era de la informatización son los datos el recurso más abundante y preciado. Estemos a la altura de nuestro potencial.

\section{Referencias bibliográficas}

AEVAl (2010). Fundamentos de Evaluación de Políticas Públicas. Ministerio de Política Territorial y Administraciones Públicas.

https://www.mptfp.gob.es/dam/es/portal/funcionpublica/evaluacion-politicas-publicas/ Documentos/Metodologias/Guia0.pdf\#page $=1$

Brugarolas, P., De la Rica, S., \& Gorjón, L. (2020). El uso de datos administrativos para la investigación. El caso de Dinamarca: un ejemplo de buenas prácticas. Fundación ISEAK. https://iseak.eu/wp-content/uploads/2020/11/El-sistema-danés-demicrodatos_DFG.pdf

De la Rica, S., Gorjón, L., \& Lizarraga, I. (2020). El contrato en prácticas en España: crónica de un desastre no anunciado. Fundación ISEAK.

https://iseak.eu/documentos/el-contratoen-practicas-en-espana-cronica-de-un-fracasono-anunciado-2/ 
Instituto para la Evaluación de Políticas Públicas (2020a). Guía de Evaluación de Diseño de Políticas Públicas. Ministerio de Política Territorial y Función Pública.

https://www.mptfp.gob.es/dam/es/portal/funcionpublica/evaluacion-politicas-publicas/ Documentos/Metodologias/Guia_de_Evaluacion_de_Diseno.pdf\#page=1

Instituto para la Evaluación de Políticas Públicas (2020b). Guía de Evaluación de Implementación de Políticas Públicas. Ministerio de Política Territorial y Función Pública. https://www.mptfp.gob.es/dam/es/portal/funcionpublica/evaluacion-politicas-publicas/ Documentos/Metodologias/Guia_de_Evaluacion_de_Implementacion.pdf\#page $=1$

Instituto para la Evaluación de Políticas Públicas (2020c). Guía de Evaluación de Resultados de Políticas Públicas. Ministerio de Política Territorial y Función Pública. https:/www.mptfp.gob.es/dam/es/portal/funcionpublica/evaluacion-politicas-publicas/ Documentos/Metodologias/Guia_de_Evaluacion_de_Resultados.pdf\#page $=1$

Instituto para la Evaluación de Políticas Públicas (2021). Evaluación de políticas públicas. Consultado el 3 de septiembre de 2021. https://www.mptfp.gob.es/portal/funcionpublica/evaluacion-politicas-publicas.html

Kleven, H., Landais, C., Posch, J., Steinhauer, A., \& Zweimüller, J. (2019). Child Penalties across Countries: Evidence and Explanations. AEA Papers and Proceedings, 109, 122-26. DOI: $10.1257 /$ pandp.20191078 